\title{
Effects of Effortful Swallow on Cardiac Autonomic Regulation
}

\author{
Lívia M. S. Gomes ${ }^{1}$ Roberta G. Silva ${ }^{1,2} \cdot$ Monique Melo $^{1} \cdot$ Nayra N. Silva ${ }^{1}$ • \\ Franciele M. Vanderlei ${ }^{3}$ - David M. Garner ${ }^{4} \cdot$ Luiz Carlos de Abreu $^{5}$. \\ Vitor E. Valenti ${ }^{1}$
}

Received: 25 August 2015/Accepted: 17 November 2015/Published online: 9 December 2015

(C) Springer Science+Business Media New York 2015

\begin{abstract}
Swallowing-induced changes in heart rate have been recently reported. However, it is not apparent the responses of heart rate variability (HRV) elicited by effortful swallow maneuver. We investigated the acute effects of effortful swallowing maneuver on HRV. This study was performed on 34 healthy women between 18 and 35 years old. We assessed heart rate variability in the time (SDNN, RMSSD, and pNN50) and frequency (HF, LF, and $\mathrm{LF} / \mathrm{HF}$ ratio) domains and, visual analysis through the Poincaré plot. The subjects remained at rest for $5 \mathrm{~min}$ during spontaneous swallowing and then performed effortful swallowing for $5 \mathrm{~min}$. HRV was analyzed during spontaneous and effortful swallowing. We found no significant differences for SDNN, pNN50, RMSSD, HF in absolute units $\left(\mathrm{ms}^{2}\right)$. There is a trend for increase of LF in absolute $(p=0.05)$ and normalized $(p=0.08)$ units during effortful swallowing. HF in normalized units reduced
\end{abstract}

Vitor E. Valenti

vitor.valenti@marilia.unesp.br

1 Centro de Estudos do Sistema Nervoso Autônomo (CESNA), Departamento de Fonoaudiologia, Faculdade de Filosofia e Ciências, UNESP, Av. Hygino Muzzi Filho, 737, Marília, SP 17.525-000, Brazil

2 Laboratório de Disfagia (LADIS), Departamento de Fonoaudiologia, Faculdade de Filosofia e Ciências, UNESP, Marília, SP, Brazil

3 Departamento de Fisioterapia, Faculdade de Ciências e Tecnologia, UNESP, Presidente Prudente, SP, Brazil

4 Cardiorespiratory Research Group, Department of Biological and Medical Sciences, Faculty of Health and Life Sciences, Oxford Brookes University, Gipsy Lane, Oxford OX3 0BP, UK

5 Laboratório de Delineamento e Escrita Científica, Faculdade de Medicina do ABC, Santo André, SP, Brazil $(p=0.02)$ during effortful swallowing and LF/HF ratio $(p=0.03)$ increased during effortful swallowing. In conclusion effortful swallow maneuver in healthy women increased sympathetic cardiac modulation, indicating a cardiac overload.

Keywords Autonomic nervous system - Cardiovascular system · Heart rate variability - Deglutition - Deglutition disorders

\section{Introduction}

The cardiovascular system is strongly influenced by the autonomic nervous system (ANS) through two important components - the sympathetic and parasympathetic nervous systems [1]. For example, there are several factors that influence cardiac autonomic regulation, such as exercise [2], chronic stress [3], and respiratory patterns [4].

Swallowing-induced tachycardia was first reported in 1926 [5] and similar mechanisms have been described [6, 7]. Some physiological mechanisms include mechanical stimulation of the left atrium by a swollen esophagus, adrenergic reflex from the esophagus and vasovagal reflex [8].

In this context, different swallowing training protocols have been applied for clinical rehabilitation in neurogenic and mechanical oropharyngeal dysphagia. Swallowing coaching protocols aim to improve quality of life of the patient, as well as severe problems such as pneumonia, malnutrition, and dehydration [9]. Improved swallowing is associated with improvement of the above mentioned complications [9]. Conversely, we hypothesize whether a swallowing training protocol, which includes effortful swallowing, acutely influences cardiac autonomic regulation. 
Heart rate variability (HRV) is well recognized as a method that assesses cardiac autonomic regulation. This method evaluates the fluctuation of the intervals between consecutive heart beats (RR intervals) [10]. Decreased levels of HRV frequently indicate inappropriate adaptation of the ANS, whereas high HRV represents good adaptation of the ANS, by characterizing a healthy individual [11].

Although a recent study reported sympathetic responses during sequential swallowing [12], it is not apparent the behavior of HRV during effortful swallowing. In addition, further mechanisms for ANS changes during swallowing training protocols is important to improve rehabilitation procedures focusing on dysphagia. Consequently, we aimed to investigate the acute effects of effortful swallowing maneuver on cardiac autonomic regulation in healthy women.

\section{Method}

\section{Population}

The subjects that participated in the study were 34 healthy women aged between 18 and 35 years old. All volunteers were informed about the procedures and the objectives of the study and gave written informed consent. All study procedures were approved by the Ethics Committee in Research of our University (No. 620-2012), and were in accordance with Resolution 196/96 National Health 10/10/ 1996.

\section{Non-inclusion Criteria}

We did not include subjects under the following conditions: body mass index (BMI) $>35 \mathrm{~kg} / \mathrm{m}^{2}$; systolic blood pressure $(\mathrm{SBP})>140 \mathrm{mmHg}$ or diastolic blood pressure (DBP) $>90 \mathrm{mmHg}$ (at rest); cardiovascular, respiratory, endocrine, and reported neurological disorders or any deglutition disorders that did not allow the volunteers to perform the procedures. Subjects under medication that influence the ANS were not included.

\section{Initial Evaluation}

Baseline information collected included: age, gender, weight, height, and body mass index (BMI). Weight was determined using a digital scale (W 200/5, Welmy, Brazil) with a precision of $0.1 \mathrm{~kg}$. Height was determined using a stadiometer (ES 2020, Sanny, Brazil) with a precision of 0.1 and $220 \mathrm{~cm}$ of extension. BMI was calculated as weight $/$ height $^{2}$, with weight in kilograms and height in meters.

\section{HRV Analysis}

Instantaneous RR intervals (RRi) were recorded with a digital telemetry system, consisting of a transmitter placed on the patient's chest and a HR monitor (Polar $^{\circledR}$ RS800CX; Polar Electro Oy, Kempele, Finland) This system detects ventricular depolarization, corresponding to the $\mathrm{R}$ wave on the electrocardiogram, at a sampling rate of $1000 \mathrm{~Hz}$ and was formerly validated [13]. They were downloaded to the Polar Precision Performance program (v.3.0, Polar Electro, Finland). The software enabled the visualization of heart rate (HR) and the extraction of a cardiac period (RR interval) file in "txt" format. Following digital filtering complemented with manual filtering for the elimination of premature ectopic beats and artifacts, $256 \mathrm{RR}$ intervals were functional for the data analysis. Only series with sinus rhythm greater than $95 \%$ were included in the study. HRV was analyzed during two time periods: spontaneous swallowing and effortful swallowing.

For HRV analysis in the frequency domain, we applied the spectral components of low frequency (LF: $0.04-015 \mathrm{~Hz}$ ) and high frequency (HF: $0.15-0.40 \mathrm{~Hz}$ ) in absolute $\left(\mathrm{ms}^{2}\right)$ and in normalized units. The spectral analysis was calculated by the Fast Fourier Transform algorithm.

Time domain analysis was achieved through the SDNN (average standard deviation of normal RR intervals), pNN50 (percentage of adjacent RR intervals lasting more difference than $50 \mathrm{~ms}$ ) and RMSSD (square root of the average square differences between normal adjacent RR intervals). For analysis of linear indices in the frequency and time domain, we applied the Kubios $\mathrm{HRV}^{\circledR}$ analysis software.

The qualitative analysis of the plot was made through the analysis of the figures formed by its attractor, which were described by Tulppo et al. [14]:

Figure in which an increase in the dispersion of RR intervals is observed with increased intervals, characteristic of a normal plot.

Small figure with beat-to-beat global dispersion without increased dispersion of RR intervals in the long term.

\section{Swallowing Training Protocol}

The swallowing training protocol was based on Lin et al. [9] and implemented according to the experience of the research team from the Laboratory of Dysphagia at the Department of Speech, Hearing and Language Therapy. The specific protocol included an effortful swallowing, which aimed to increase swallowing strength, improve 
coordination and decrease the posterior movement of the tongue base.

\section{Experimental Protocols}

Data collection was undertaken in the same sound-proofed room for all volunteers with the temperature between 21 and $26^{\circ} \mathrm{C}$; relative humidity between 40 and $60 \%$. Volunteers were instructed not to drink alcohol and/or caffeine for $24 \mathrm{~h}$ before evaluation, with a light meal at least $2 \mathrm{~h}$ before experimentation. Datasets were collected on an individual basis, between 18:00 and 21:00 to standardize the protocol. All procedures necessary for the data collection were explained on an individual basis and the subjects were instructed to remain at rest and avoid talking during the collection.

After the initial evaluation the heart monitor belt was then placed over the thorax as beforehand.

The volunteers were instructed to remain $5 \mathrm{~min}$ at rest with spontaneous swallowing, after the initial $5 \mathrm{~min}$ they performed the swallowing training protocol for $5 \mathrm{~min}$. HRV was compared between spontaneous swallowing and during swallowing training protocol.

\section{Statistical Analysis}

Standard statistical methods were applied for the calculation of means and standard deviations. Normal Gaussian distribution of the data was verified by the Shapiro-Wilk goodness-of-fit test $(z$ value $>1.0)$. For parametric distributions, we applied paired Student $t$ test and, for nonparametric distributions we applied Wilcoxon test. HRV indices were compared between spontaneous and effortful swallowing. Differences were considered significant when the probability of a Type I error was $<5 \%(p<0.05)$. We used the Software Biostat ${ }^{\circledR} 2009$ Professional 5.8.4.

\section{Results}

Table 1 illustrates the values for baseline heart rate (HR), mean RR intervals, weight, height, systolic, and diastolic arterial pressure and body mass index (BMI) of the volunteers.

Figure 1 shows mean and standard deviation of the time domain indices of HRV during spontaneous swallowing and effortful swallowing. We observed no significant differences for SDNN, pNN50, and RMSSD indices between the two conditions.

Mean and standard deviation of the frequency domain indices of HRV during spontaneous swallowing and effortful swallowing are presented in Fig. 2. The frequency domain indices are calculated through Fast Fourier
Table 1 Mean and standard deviation of baseline

\begin{tabular}{lc}
\hline Variable & Value \\
\hline Age (years) & $20.4 \pm 1.8$ \\
Weight $(\mathrm{kg})$ & $60 \pm 10.1$ \\
Height $(\mathrm{m})$ & $1.64 \pm 0.06$ \\
BMI $\left(\mathrm{kg} / \mathrm{m}^{2}\right)$ & $25.4 \pm 3.5$ \\
Mean HR $(\mathrm{bpm})$ & $85.5 \pm 10$ \\
Mean RR (ms) & $715.4 \pm 88$ \\
SAP (mmHg) & $10.9 \pm 1$ \\
DAP (mmHg) & $7.5 \pm 1$ \\
\hline
\end{tabular}

$\overline{H R}$ heart rate, $S A P$ systolic and $D A P$ diastolic arterial pressure, Mean $R R$ mean RR interval, $B M I$ weight, height and body mass index of the volunteers, $m$ meters, $\mathrm{kg}$ kilograms, $\mathrm{bpm}$ beats per minute, $m s$ milliseconds, $\mathrm{mmHg}$ millimeters of mercury

Transform algorithm and we noted no significant change in $\mathrm{HF}$ in absolute units $\left(\mathrm{ms}^{2}\right)$ and a trend for increase in LF in absolute and normalized units during effortful swallowing. Alternatively, HF in normalized units reduced and LF/HF ratio increased during effortful swallowing.

The visual analysis of HRV behavior during spontaneous swallowing and effortful swallowing through Poincaré plot from one subject during spontaneous swallowing (A) and effortful swallowing (B) is shown in Fig. 3. In the Poincaré plot, figure that illustrates an increase in the distribution of RR intervals indicate a healthy plot. Alternatively, small figure with beat-to-beat global dispersion with no increased distribution of RR intervals in the long-term indicates impaired health.

\section{Discussion}

We endeavored to investigate the acute effects of effortful swallowing on cardiac autonomic regulation in healthy women. As a key discovery, it was observed that cardiac sympathetic regulation increased during effortful swallowing, indicating cardiac overload in this population.

Based on our data, time domain indices of HRV were not changed during effortful swallowing. However, the frequency domain analysis indicated that the sympathetic cardiac component of HRV was increased during effortful swallowing, since LF/HF was enhanced.

Sympathetic activation induces tachycardia [1, 15] and as recently reported, swallowing-induced tachyarrhythmia in pathological situation [6]. This mechanism is suggested to be an irregular automaticity complex through re-entrant tachycardia [16]. Another study illustrated that swallowing salbutamol caused swallowing-induced atrial tachyarrhythmia likely due to a beta-agonist salbutamol effect, which is originated in the esophagus while swallowing triggering adrenergic reflexes [8]. 

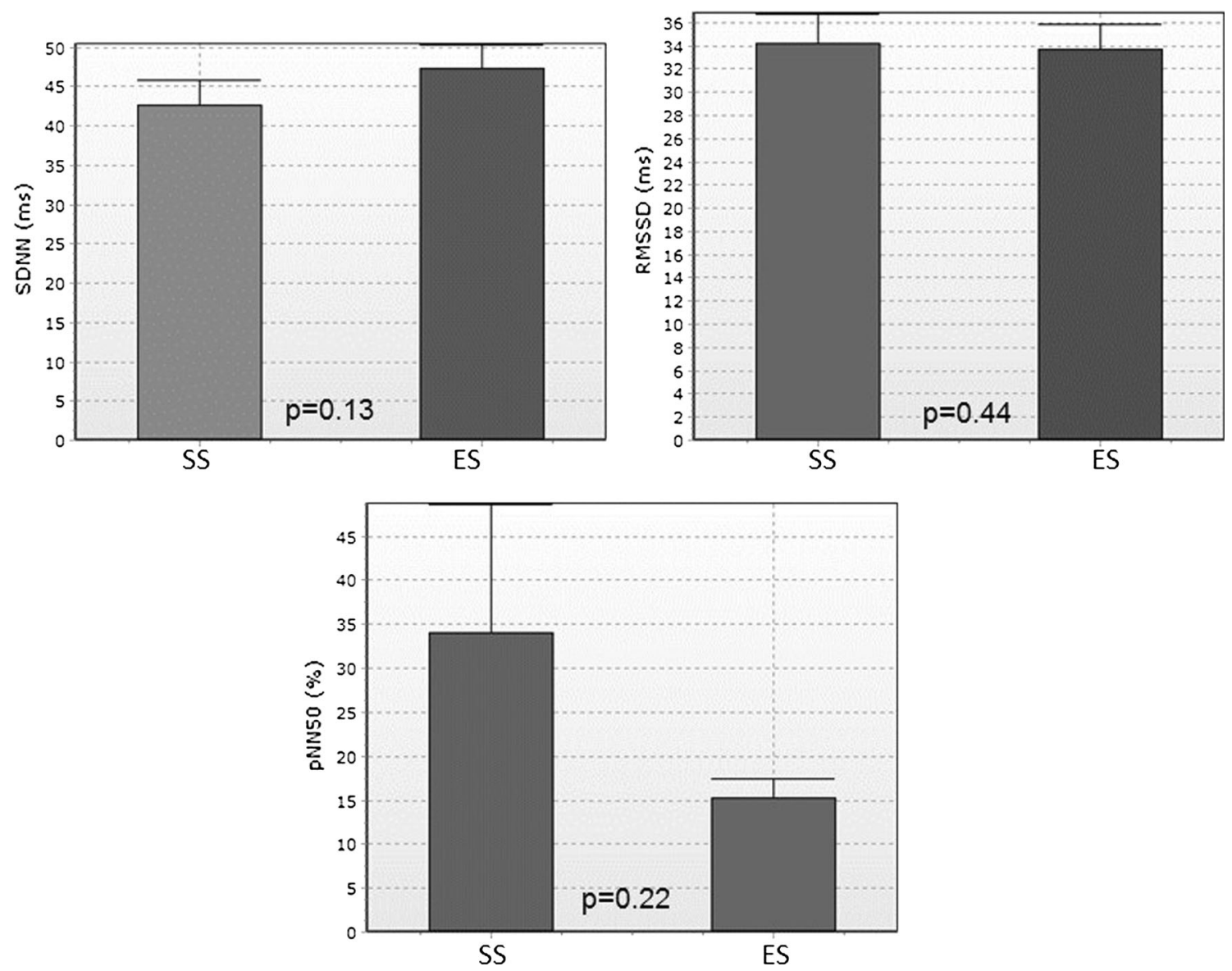

Fig. 1 Time domain indices of HRV during spontaneous (SS) and effortful swallowing (HD). SDNN standard deviation of normal-tonormal $\mathrm{R}-\mathrm{R}$ intervals, RMSSD root-mean square of differences

In this situation, our investigation illustrated that the LF/ HF ratio increased during effortful swallowing - this index represents the sympathovagal balance [10]. Although swallowing-induced tachyarrhythmia is a uncommon event [16], it supports our information that effortful swallowing may be involved in sympathetic activation.

Furthermore, the visual analysis of the Poincare Plot revealed changes in HRV during effortful swallowing, with a lower beat-to-beat dispersion of RR intervals and a lower dispersion of RR intervals over the long term during effortful swallowing. This finding indicates that the global $\mathrm{HRV}$ is reduced during effortful swallowing.

According to our study, the HF in normalized units significantly reduced during effortful swallowing, indicating parasympathetic decrease. The HF band is considerably influenced by breath [1] and the swallowing training protocol presents a substantial effect on respiratory rate and intrathoracic pressure. Altogether, we believe that the parasympathetic component of HRV is not significantly involved in HRV changes during effortful swallowing between adjacent normal RR intervals in a time interval. SDNN/ RMSSD ration; pNN50: percentage of adjacent RR intervals with a difference of duration $>50 \mathrm{~ms}, m s$ milliseconds

while the sympathetic nervous system is hypothesized to present stronger influence.

We suggest that respiratory parameter is a major factor involved in cardiac autonomic changes induced by swallowing because when the subjects are instructed to perform effortful swallowing they need to be suddenly interrupted. Previous studies reported that controlled breaths cause different effects on autonomic neural outflow [4, 17]. A standard example is the respiratory sinus arrhythmia, which is a physiological change in heart rate oscillations associated with respiration rate $[4,18]$.

In this manner, a previous study instructed volunteers to perform different breaths and the ANS was analyzed; they observed that during hyperventilation muscle sympathetic nerve activity increased and RR interval was reduced. Moreover, respiratory sinus arrhythmia significantly influenced HRV in healthy subjects and patients with chronic respiratory disease [17].

In order to standardize the HRV parameters for all subjects, we undertook all procedures between 18:00 and 

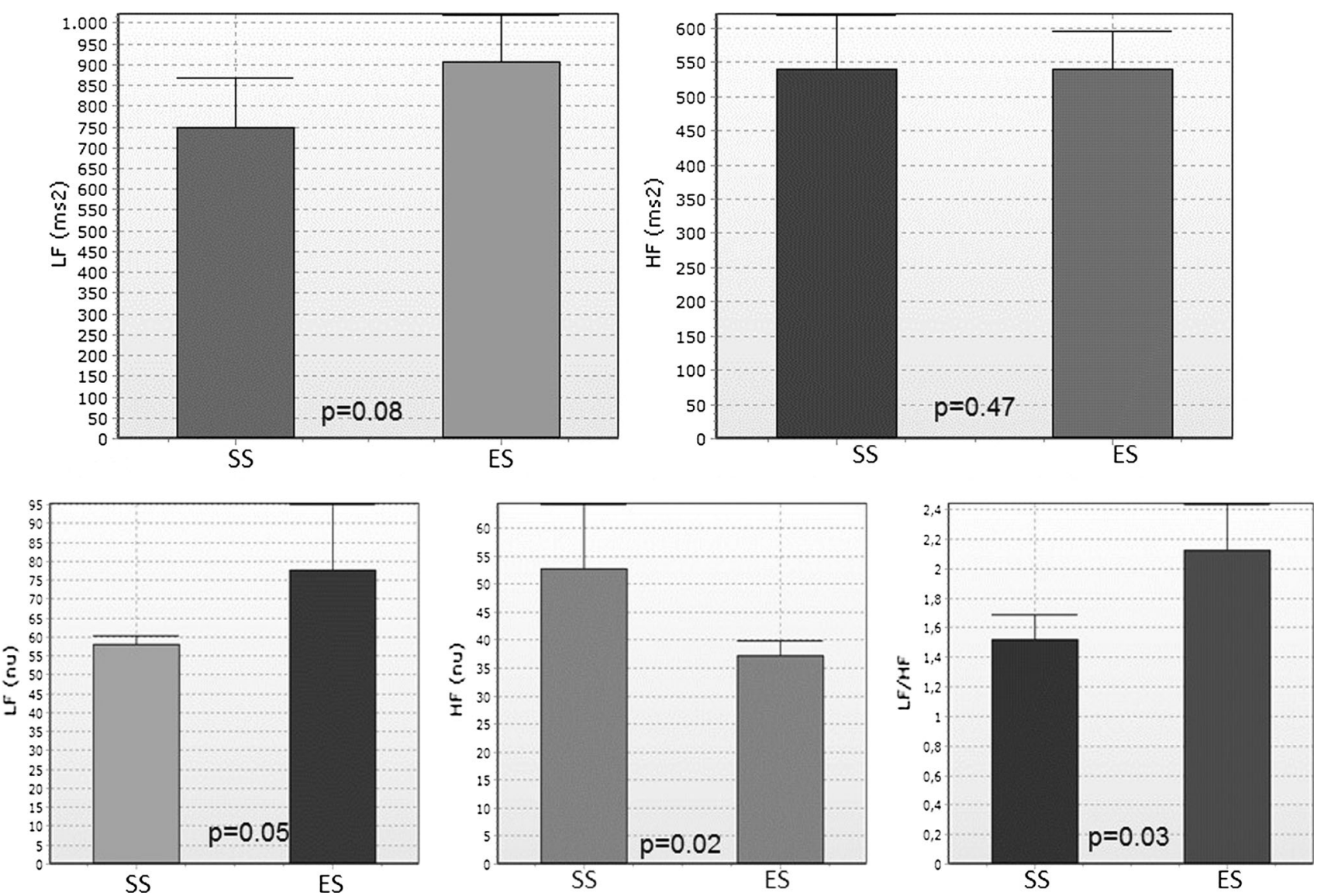

Fig. 2 Frequency domain indices of HRV during spontaneous (SS) and effortful swallowing (ED). $L F$ low frequency, $H F$ high frequency, $L F /$ $H F$ low frequency/high frequency ratio, $m s$ milliseconds, $n u$ normalized units

21:00. This is important because melatonin presents association with HRV [19] and intensely influences temperature, heart rate and other physiological responses regulated by the autonomic nervous system [20].

In this light, Numata et al. [21] investigated circadian changes of influence of swallowing on respiratory sinus arrhythmia. The authors found that the smallest RR interval during breath with swallowing was higher in the morning than in the afternoon or evening. Furthermore, the amplitude of the respiratory sinus arrhythmia during breathing with swallowing was higher in the morning than in the evening, supporting an important influence of circadian rhythm on the interaction between swallowing and autonomic nervous system.

Some points in our study are worth highlighting. We analyzed only healthy women because sexual hormones have potential effects on $\operatorname{HRV}[22,23]$ and laryngeal muscle fibers [24]. We did not focus on a specific substance during swallowing. We aimed to investigate a training swallowing protocol with no substance because that protocol has been used for patients with dysphagia [9]. The effortful swallowing used in our protocol aims to increase muscle strength, improve coordination, and reduce the posterior movement of the tongue base [9]. However, clinicians were not concerned about the possible effects of this rehabilitation protocol on cardiac events.

Some points are worth highlighting in our study. We investigated only 34 healthy women; so we encourage additional studies in patients with cardiovascular and swallowing disorders. Moreover, it is also relevant to mention that there are sexual hormones-related effects on cardiac autonomic regulation [25]. In this manner, we should not extrapolate our findings to male subjects.

Standard deviations were large; and this possibly reduced the chances to attain significance between some indices, such as pNN50, RMSSD, and SDNN that fit time domain indices analysis of HRV. We believe that it is due to the absence of swallowing muscle contraction measurement during the protocols, which is a restriction of our study. If we quantified muscle contraction, we would be able to better regulate the swallowing strength of each volunteer.

Our data indicated that effortful swallowing caused cardiac overload through increase in the sympathetic 

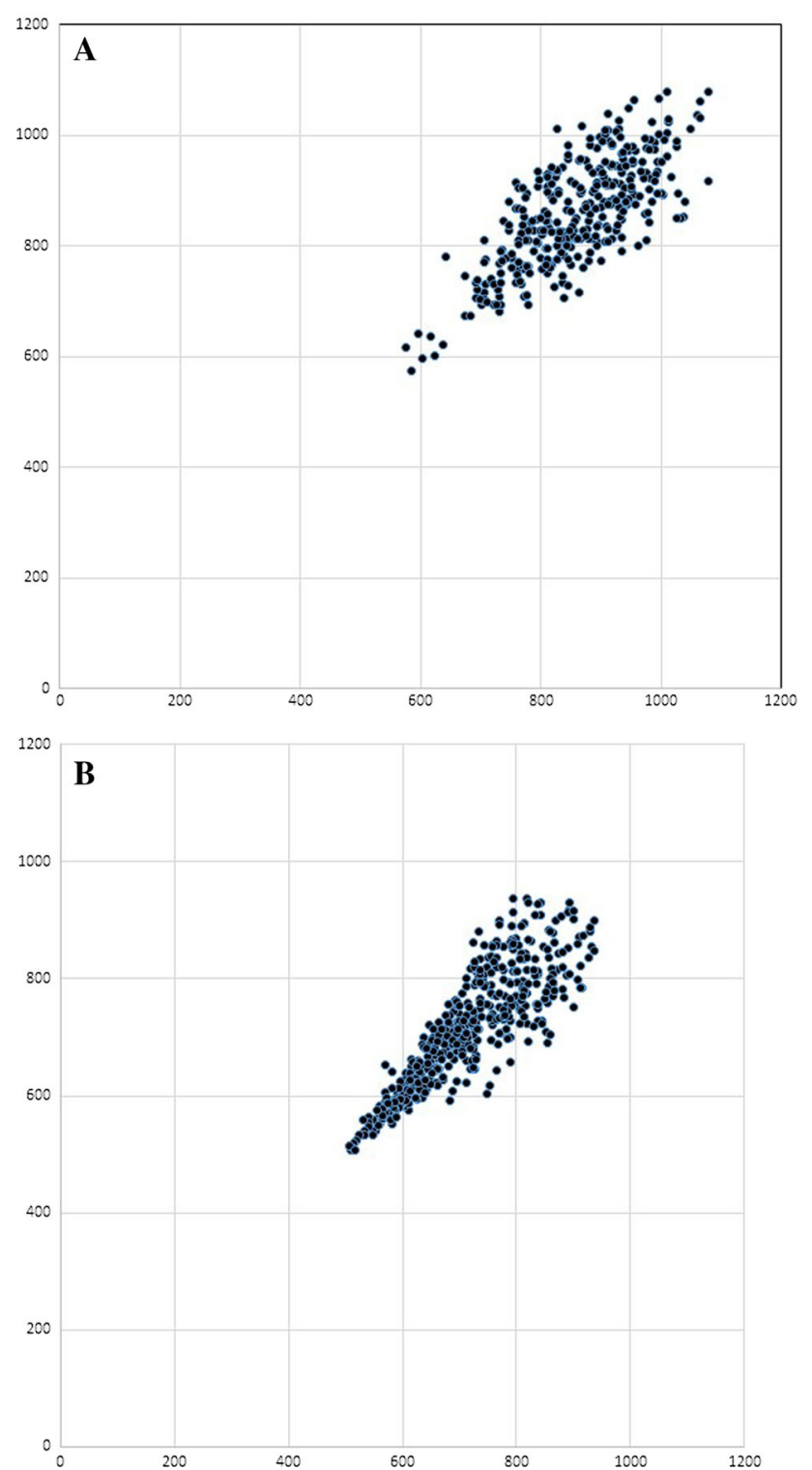

Fig. 3 Visual pattern of the Poincare plot observed in one subject during spontaneous (a) and effortful swallowing (b)

cardiac regulation in healthy women. Consequently, we suggest further studies in clinically relevant patients to better understand this mechanism.

\section{Conclusion}

A swallowing training protocol based on effortful swallowing acutely increased sympathetic cardiac modulation in healthy women. Our data suggests additional studies to investigate this mechanism in patients with swallowing and cardiac events.

\section{Compliance with Ethical Standards}

Conflict of Interest There is no conflict of interest.

\section{References}

1. Vanderlei LC, Pastre CM, Hoshi RA, Carvalho TD, Godoy MF. Basic notions of heart rate variability and its clinical applicability. Rev Bras Cir Cardiovasc. 2009;24:205-17.

2. Moreno IL, Pastre CM, Ferreira C, Abreu LC, Valenti VE, Vanderlei LC. Effects of an isotonic beverage on autonomic regulation during and after exercise. J Int Soc Sports Nutr. 2013;10:2.

3. Kovács L, Kézér FL, Jurkovich V, Kulcsár-Huszenicza M, Tözsér J. Heart rate variability as an indicator of chronic stress caused by lameness in dairy cows. PLoS ONE. 2015;10:e0134792.

4. Badra LJ, Cooke WH, Hoag JB, Crossman AA, Kuusela TA, Tahvanainen KU, et al. Respiratory modulation of human autonomic rhythms. Am J Physiol Heart Circ Physiol. 2001;280:H2674-88.

5. Sakai D, Mori F. Uber einen Fall von sog "Schlucktachycardie". Z Gesamte Exp Med. 1926;50:106.

6. Tanoue K, Sonoda M, Yamashita E, Tanaka H, Nuruki N. Swallowing-induced atrial tachyarrhythmia triggered by solid foods. Circulation. 2014;130:e113-5.

7. Yokoshiki H, Mitsuyama H, Watanabe M, Tsutsui H. Swallowing-induced multifocal atrial tachycardia originating from right pulmonary veins. J Electrocardiol. 2011;44:395.e1-5.

8. Tandeter H, Kobal S, Katz A. Swallowing-induced atrial tachyarrhythmia triggered by salbutamol: case report and review of the literature. Clin Cardiol. 2010;33:e116-20.

9. Lin LC, Wang SC, Chen SH, Wang TG, Chen MY, Wu SC. Efficacy of swallowing training for residents following stroke. J Adv Nurs. 2003;44:469-78.

10. Task force of the European Society of Cardiology and the North American Society of Pacing and Electrophysiology. Heart rate variability: standards of measurement, physiological interpretation and clinical use. Circulation. 1996;93:1043-65.

11. Pumprla J, Howorka K, Groves D, Chester M, Nolan J. Functional assessment of heart rate variability: physiological basis and practical applications. Int J Cardiol. 2002;84:1-14.

12. Arıcı Ş, Gürgör N, Seçil Y, İncesu TK, Özdemirkıran T, Çe P, et al. Sympathetic skin responses in adult humans during sequential swallowing. Neurophysiol Clin. 2013;43:11-7.

13. Barbosa MPR, Silva NT, Azevedo FM, Pastre CM, Vanderlei LC. Comparison of Polar ${ }^{\circledR}$ RS800G3 ${ }^{\mathrm{TM}}$ heart rate monitor with Polar ${ }^{\circledR} \mathrm{S} 810 \mathrm{i}^{\mathrm{TM}}$ and electrocardiogram to obtain the series of RR intervals and analysis of heart rate variability at rest. Clin Physiol Funct Imaging. 2014. doi:10.1111/cpf.12203.

14. Tulppo MP, Mäkikallio TH, Seppänen T, Laukkanen RT, Huikuri HV. Vagal modulation of heart rate during exercise: effects of age and physical fitness. Am J Physiol. 1998;2:424-9.

15. Abreu LC. Heart rate variability as a functional marker of development. J Hum Growth Develop. 2012;22:279-81.

16. Nakagawa H, Scherlag BJ, Patterson E, Ikeda A, Lockwood D, Jackman WM. Pathophysiologic basis of autonomic ganglionated plexus ablation in patients with atrial fibrillation. Heart Rhythm. 2009;6:S26-34.

17. Reis MS, Deus AP, Simões RP, Aniceto IA, Catai AM, BorghiSilva A. Autonomic control of heart rate in patients with chronic cardiorespiratory disease and in healthy participants at rest and 
during a respiratory sinus arrhythmia maneuver. Rev Bras Fisioter. 2010;14:106-13.

18. Carrasco-Sosa S, Gaitán-González MJ, González-Camarena R, Yáñez-Suárez O. Baroreflex sensitivity assessment and heart rate variability: relation to maneuver and technique. Eur $\mathrm{J}$ Appl Physiol. 2005;95:265-75.

19. Boudreau P, Dumont G, Kin NM, Walker CD, Boivin DB. Correlation of heart rate variability and circadian markers in humans. Conf Proc IEEE Eng Med Biol Soc. 2011;2011:681-2.

20. Cajochen C, Münch M, Kobialka S, Krauchi K, Steiner R, Oelhafen $\mathrm{P}$, et al. High sensitivity of human melatonin, alertness, thermoregulation, and heart rate to short wavelength light. J Clin Endocrinol Metab. 2005;90:1311-6.

21. Numata T, Kishida Y, Jimbo Y, Kotani K. Circadian changes of influence of swallowing on heart rate variability with respiratoryphase domain analysis. Conf Proc IEEE Eng Med Biol Soc. 2013;2013:5377-80.

22. Poliwczak AR, Tylińska M, Broncel M. Effect of short-term testosterone replacement therapy on heart rate variability in men with hypoandrogen-metabolic syndrome. Pol Arch Med Wewn. 2013;123:467-73.
23. Tenan MS, Brothers RM, Tweedell AJ, Hackney AC, Griffin L. Changes in resting heart rate variability across the menstrual cycle. Psychophysiology. 2014;51:996-1004.

24. Watson JT, Robertson J, Sachdev U, Kelley DB. Laryngeal muscle and motor neuron plasticity in Xenopus laevis: testicular masculinization of a developing neuromuscular system. J Neurobiol. 1993;24:1615-25.

25. Thayer JF, Sollers JJ, Friedman BH, Koenig J. Gender differences in the relationship between resting heart rate variability and 24-hour blood pressure variability. Blood Press. 2015;29:1-5.

Roberta G. Silva $\mathrm{PhD}$

Franciele M. Vanderlei $\mathrm{PhD}$

Luiz Carlos de Abreu PhD

Vitor E. Valenti $\mathrm{PhD}$ 